\title{
Patterns of symptoms before a diagnosis of first episode psychosis: a latent class analysis of UK primary care electronic health records
}

Ying Chen ${ }^{1 *}$, Saeed Farooq ${ }^{1}$, John Edwards ${ }^{1}$, Carolyn A. Chew-Graham ${ }^{1}$, David Shiers ${ }^{1,2,3}$, Martin Frisher ${ }^{4}$, Richard Hayward ${ }^{1}$, Athula Sumathipala ${ }^{1}$ and Kelvin P. Jordan ${ }^{1}$

\begin{abstract}
Background: The nature of symptoms in the prodromal period of first episode psychosis (FEP) remains unclear. The objective was to determine the patterns of symptoms recorded in primary care in the 5 years before FEP diagnosis.

Methods: The study was set within 568 practices contributing to a UK primary care health record database (Clinical Practice Research Datalink). Patients aged 16-45 years with a first coded record of FEP, and no antipsychotic prescription more than 1 year prior to FEP diagnosis $(n=3045)$ was age, gender, and practice matched to controls without FEP $(n=12,180)$. Fifty-five symptoms recorded in primary care in the previous 5 years, categorised into 8 groups (mood-related, 'neurotic', behavioural change, volition change, cognitive change, perceptual problem, substance misuse, physical symptoms), were compared between cases and controls. Common patterns of symptoms prior to FEP diagnosis were identified using latent class analysis.

Results: Median age at diagnosis was 30 years, 63\% were male. Non-affective psychosis (67\%) was the most common diagnosis. Mood-related, 'neurotic', and physical symptoms were frequently recorded ( $>30 \%$ of patients) before diagnosis, and behavioural change, volition change, and substance misuse were also common (> 10\%). Prevalence of all symptom groups was higher in FEP patients than in controls (adjusted odds ratios 1.33-112). Median time from the first recorded symptom to FEP diagnosis was 2-2.5 years except for perceptual problem (70 days). The optimal latent class model applied to FEP patients determined three distinct patient clusters: 'no or minimal symptom cluster' (49\%) had no or few symptoms recorded; 'affective symptom cluster' (40\%) mainly had mood-related and 'neurotic' symptoms; and 'multiple symptom cluster' (11\%) consulted for three or more symptom groups before diagnosis. The multiple symptom cluster was more likely to have drug-induced psychosis, female, obese, and have a higher morbidity burden. Affective and multiple symptom clusters showed a good discriminative ability (C-statistic 0.766; sensitivity $51.2 \%$ and specificity $86.7 \%$ ) for FEP, and many patients in these clusters had consulted for their symptoms several years before FEP diagnosis.
\end{abstract}

Conclusions: Distinctive patterns of prodromal symptoms may help alert general practitioners to those developing psychosis, facilitating earlier identification and referral to specialist care, thereby avoiding potentially detrimental treatment delay.

Keywords: First episode psychosis, Symptom cluster, General practice, Medical record research, Latent class analysis, Epidemiology

\footnotetext{
* Correspondence: y.chen1@keele.ac.uk

${ }^{1}$ School of Primary, Community and Social Care, Keele University, Keele ST5

$5 B G$, UK

Full list of author information is available at the end of the article
}

(c) The Author(s). 2019 Open Access This article is distributed under the terms of the Creative Commons Attribution 4.0 International License (http://creativecommons.org/licenses/by/4.0/), which permits unrestricted use, distribution, and reproduction in any medium, provided you give appropriate credit to the original author(s) and the source, provide a link to the Creative Commons license, and indicate if changes were made. The Creative Commons Public Domain Dedication waiver (http://creativecommons.org/publicdomain/zero/1.0/) applies to the data made available in this article, unless otherwise stated. 


\section{Background}

There is often a substantial gap between the first presentation of symptoms and subsequent diagnosis of a first episode psychosis (FEP) leading to a delay in treatment and worse outcomes [1]. The average duration of untreated psychosis (DUP), the period between the first onset of psychotic symptoms and treatment, has been reported to be over a year [2-4]. Several independent meta-analyses have provided evidence for the association between long DUP and poor outcome. Marshall et al. included 26 studies involving prospective cohorts with over 4000 participants. This meta-analysis revealed significant associations between long DUP and poor outcomes in symptomatic and functional domains at 6 and 12 months after diagnosis, which were independent of co-morbidity [5]. Perkins et al. included 44 studies involving 5491 participants and demonstrated that longer DUP was associated with less response to antipsychotic medication [1]. Penttilä et al. included 33 studies with a mean follow-up of 8.1 years and concluded that long DUP was associated with poor general symptomatic outcome, more severe positive and negative symptoms, lesser likelihood of remission, and poor social functioning and global outcome in the long term [4]. Reducing DUP has become the primary aim of modern psychiatric services for patients with a FEP.

Initiatives to shorten DUP, however, have largely been unsuccessful [6]. This is partly due to the fact that the nature of symptoms in the prodromal period remains unclear. In a systematic review, Anderson et al. explored the nature of the pathway to care for patients experiencing a FEP. Almost all studies in this review explored the sex, socio-economic, or ethnic determinants of the pathways to care. The authors commented that the nature of the pathway to care, which includes understanding the symptoms and presentations in the period before the diagnosis of FEP, is crucial in understanding the delay in contact with services [7]. Some studies have examined the help-seeking behaviour in terms of psychological processes such as locus of control or type of symptoms leading to contacts with health professionals before FEP diagnosis [8, 9]. Platz et al. found that patients with psychotic symptoms more often contacted mental health professionals, whereas patients with insidious and more unspecific features more frequently contacted general practitioners (GPs) [9]. Anderson et al. also found in their review that in 13 of 21 studies which examined pathways to care, the first contact for most patients was with a physician (including all 3 UK studies). However, the referral source to psychiatric services for the greatest proportion of patients was emergency services in 9 of 22 studies which examined such topic (including 2 of 4 UK studies) [7]. In addition, data from the UK suggested that individuals referred by a home treatment team or the emergency service had the lowest DUP [10]. These probably reflected the lack of identification of the symptoms suggestive of FEP in routine care settings, including primary care services. This is despite the evidence that contacts with primary care may increase prior to the diagnosis of FEP: a Danish registerbased study suggested an increase in primary care contacts several years prior to the diagnosis of schizophrenia [11]. A UK study also demonstrated that in general, higher frequency of GP contact before the onset of psychosis was associated with shorter DUP [8]. However, a small study in Switzerland found that patients consulted GPs with insidious features which were not recognised by GPs as being an indication of FEP, hence causing delays in referral to specialised services, a diagnosis made, and treatment initiated [9].

Patterns of consultation and presentation of symptoms in primary care before the diagnosis of psychosis have not been fully studied. Identification of these symptoms, including psychological and physical and substance misuse, may help to identify patients earlier, thus helping to reduce unacceptably long DUP. The objective of this study was to determine common symptoms and patterns of symptomatology presented to primary care prior to diagnosis of FEP. This would help inform the need for better and more targeted risk management by clinicians when patients present with symptoms suggestive of FEP.

\section{Methods \\ Setting}

The study was set within the Clinical Practice Research Datalink (CPRD). CPRD is a pseudo-anonymised database of routinely recorded general practice information from over 10 million patients registered with over 670 UK primary care practices [12]. Diagnosis of mental and behavioural disorders recorded in CPRD has been validated using internal (i.e. manual review of diagnostic algorithm) and external (i.e. questionnaire to GP, record request to GP, questionnaire and record request to GP, comparison of rates) methods by 20 studies [13]. It was also shown that the rates of GP recorded severe mental illness in UK primary care were broadly comparable to incidence rates from previous epidemiological studies of severe mental illness in the UK [14].

\section{Study population}

We included patients aged 16-45 years with a first coded record of a FEP (defined as affective, non-affective, druginduced, and pregnancy-related psychoses) between 1 April 2005 (1 year after the introduction of the Quality and Outcomes Framework (QOF) into UK primary care [15]) and 31 December 2016, who had been registered at a practice contributing to CPRD for at least 5 years before the recorded FEP, and had not received an antipsychotic 
prescription more than 1 year prior to FEP. The rationale for the antipsychotic prescription criterion was that treatment may commence before a formal diagnosis has been entered onto the clinical system, but if treatment is recorded more than a year before diagnosis, this would suggest ongoing active treatment but inadequate diagnostic recording in primary care. Patients with a recorded code for Parkinson's disease or dementia in the 5 years prior to FEP were ineligible. Patients with a coded record of psychosis in remission during the 5-year period were also excluded.

A control group was identified, matched 4:1 [16] by year of birth (age), gender, and practice, to the FEP group. The controls had no recorded consultations for FEP, psychosis in remission or antipsychotic prescription, and had at least 5 years prior registration before the index date (which was the diagnosis date of FEP for their matched case).

\section{Exposure}

Recorded symptoms potentially related to FEP in the 5 years prior to the index date in both patients with FEP and controls were identified and categorised into eight groups. These symptom groups were determined through a review of the literature [17-19] and consensus of a consultant in psychiatry (SF) and two general practitioners with electronic health records research experience (JE, RH). In general, the final eight groups were based on the concept of Yung and McGorry as a foundation [17] and included psychological, substance-related, and physical symptoms (Table 1).

\section{Covariates}

Covariates thought to be potentially associated with symptoms/psychosis and recorded in CPRD were year of the index date, age, gender, geographical region, smoking status, alcohol consumption, body mass index (BMI), specific co-morbidities, total morbidity burden, and the frequency of GP consultation.

At the practice level, the geographical region was recorded by CPRD as 1 of 13 regions in the UK [12]. In this study, these regions were further summarised into London, South England (South West, South Central, South East Coast), Midlands and East England (East Midlands, West Midlands, East of England), North England (North East, North West, Yorkshire, and the Humber), Wales, Scotland, and Northern Ireland.

Smoking and alcohol information was classified as ever smoked/drunk alcohol, never, or missing, based upon the data recorded before the index date. The BMI value used was the most recent record before the index date and was grouped into $<18.5$ (underweight), $\geq 18.5$ and $<25$ (normal), $\geq 25$ and $<30$ (overweight), $\geq 30 \mathrm{~kg} / \mathrm{m}^{2}$ (obese), or missing. Sensitivity analyses in patients with and without missing data on smoking, alcohol drinking, and BMI are briefly shown in Additional file 1. Findings were similar in both groups.

Physical comorbidities often co-exist in people with a mental health problem [20]. Common conditions previously found to be associated with psychosis were included in this study. Candidate conditions included diabetes, ischaemic heart disease, asthma, chronic obstructive pulmonary disease, inflammatory diseases (rheumatoid arthritis, gout, polymyalgia rheumatic, inflammatory bowel disease, systemic lupus erythematosus, spondyloarthritis), hypertension, chronic kidney disease, musculoskeletal pain (back, foot/ankle, hand/wrist, hip, knee, neck, shoulder), and injury and major trauma. These conditions were identified during the 5-year period prior to the index date.

Table 1 Groups of prodromal symptoms in psychosis

\begin{tabular}{|c|c|}
\hline Symptom group & Individual symptoms ${ }^{\dagger}$ included in each group \\
\hline \multicolumn{2}{|l|}{ Psychological symptom } \\
\hline $\begin{array}{l}\text { Mood-related } \\
\text { symptom }\end{array}$ & Depression, anhedonia, guilt, mood swings, suicidal/self-harm ideation or behaviour \\
\hline 'Neurotic' symptom & Neuroses, anxiety, irritability and anger, restlessness, worrying thoughts \\
\hline Behavioural change & Deterioration, social withdrawal, impulsivity, reduced self-esteem, aggressive and disruptive behaviour, odd behaviour \\
\hline Change in volition & Apathy (loss of drive), tiredness/fatigue (loss of energy), boredom (loss of interest) \\
\hline Perceptual problem & Hallucinations, delusions, illusions \\
\hline Cognitive change & $\begin{array}{l}\text { Disturbance of attention, concentration/preoccupation difficulties, cognitive/memory impairment, thought disorder/ } \\
\text { blocking }\end{array}$ \\
\hline Substance misuse & $\begin{array}{l}\text { Opioids, alcohol, cannabis, hypnotic, cocaine, amphetamine, glue, tobacco, hallucinogen, ecstasy, antidepressant, solvent, } \\
\text { other/multiple stimulant, general (codes without specific substance) }\end{array}$ \\
\hline Physical symptom & $\begin{array}{l}\text { Speech abnormality, sleep disturbance, loss of weight, poor appetite, dryness of the mouth, dysphagia, hyperventilation, } \\
\text { muscle tension, epigastric discomfort, palpitations, shortness of breath, excessive wind, decreased libido, menstrual } \\
\text { problem (in females), failure of erection (in males) }\end{array}$ \\
\hline
\end{tabular}

${ }^{\dagger}$ Total number of individual symptoms studied, $n=55$ 
In addition, prescriptions for drugs recorded under different British National Formulary (BNF) chapters in the 5-year period prior to the index date was used as a surrogate measure of the total morbidity burden, which has been shown to be as predictive of health outcomes as more complicated comorbidity measures [21].

The frequency of GP consultation was determined annually over the 5 years prior to the index date.

\section{Codes and identification}

In UK primary care, problems, including symptoms and diagnoses, are generally recorded using the 'Read' system of codes [22]. Diagnosis of FEP and prodrome symptoms were identified by the use of a Read code list developed through consensus of clinical researchers (SF, JE, and $\mathrm{RH}$, see Additional file 2). Identification of co-morbid conditions used prior established Read code lists within the research institute.

Antipsychotic medications were defined as medications under BNF chapter 4.2 (by JE, see Additional file 2).

\section{Statistical analysis}

The recorded consultation prevalences of each individual symptom and symptom group (at least one recorded symptom from a group) in patients with FEP and matched controls during the 5-year prior to the index date were determined. Conditional logistic regression was used to analyse the associations of individual symptoms and symptom groups with FEP in separate models, adjusted for the other covariates and reported using odds ratios (ORs) with 95\% confidence intervals (CIs). Cluster-robust variance estimators were used to take into account clustering by practice.

For patients with FEP, for each symptom group, the time interval between the first recorded symptom (if any) and diagnosis was determined. We also identified the earliest record (if any) of symptoms, regardless of symptom group, for each patient.

Within patients with FEP, latent class analysis (LCA) was used to determine common patterns of symptoms, at the level of the symptom groups, presented to primary care over the 5-year period before FEP diagnosis. The LCA clustered patients with FEP into distinct groups based on their pattern of prior recorded symptoms across the 8 symptom groups, with each patient allocated to one cluster [23]. We used $L^{2}$ statistics with bootstrap $p$ values, Bayes Information Criterion (BIC), and Consistent Akaike's Information Criterion (CAIC) to determine the optimal model, i.e. the optimal number of clusters. Latent GOLD (version 4.5) was used to perform the analyses, using both the estimation-maximisation and NewtonRaphson algorithms to estimate model parameters. One thousand different random starting values were used, each of which included 100 iterations. Bootstrap $p$ values based on 500 replications were determined to assess the model fit based on the $L^{2}$ statistics. The optimal number of clusters is where the $p$ value becomes non-significant at the desired significance level (5\%). For the BIC and CAIC, the optimal model is the model with the smallest information criterion values. Patients were allocated to clusters based on their posterior probabilities of belonging to each cluster. A mean posterior probability $\geq 0.7$ for patients allocated to a cluster was considered acceptable [24]. Based on the optimal model, the identified clusters were compared at diagnosis on patient characteristics (psychosis subtype, age, gender, geographical region, smoking, alcohol drinking, BMI, morbidity burden, frequency of consultation). The proportions of patients in each cluster with pre-recorded symptoms 4 , 3,2 , and 1 year before diagnosis were identified.

We also mapped each control to the clusters that had been identified in cases based on their recorded symptoms during the 5 years before the index date. Then, in the case-control setting, a conditional logistic regression model containing symptom cluster as an independent variable was used to determine the discriminative ability of these clusters to predict a FEP diagnosis, assessed using C-statistic with 95\% CIs. A value $\geq 0.75$ was considered to indicate good discrimination.

All analyses were performed using STATA/MP 15 if not stated elsewhere.

\section{Patient and public involvement}

The research findings were discussed with a convenor of a local support group ('Hear Our Voices', North Staffordshire, England) that aims to give a voice to people with a mental health problem.

\section{Results \\ Patient characteristics}

Three thousand forty-five FEP patients (63\% male, median age 30) and 12,180 age-, gender-, and practicematched controls were included in the analysis from 568 general practices. FEP patients were diagnosed most commonly with non-affective psychosis (67\%), followed by drug-induced $(22 \%)$, affective $(10 \%)$, and pregnancyrelated (1\%) psychoses (Table 2).

FEP patients were more likely than controls to smoke and had a lower BMI. Increased morbidity burden was observed in FEP patients (median of 4 different prescriptions in the previous 5 years versus 3 in the controls). The median number of GP consultations for FEP patients was more than double those of controls during the 5-year period before the index date. FEP patients increased from a median of 6 consultations in the time period 49-60 months before diagnosis to 17 
Table 2 Participant demographic and clinical characteristics at the index date

\begin{tabular}{|c|c|c|c|}
\hline & FEP patients $(n=3045)$ & Matched participants $(n=12,180)$ & $p$ value \\
\hline \multicolumn{4}{|l|}{ Type of psychosis, n (\%) } \\
\hline Non-affective & $2036(66.9)$ & NA & \\
\hline Drug-induced & $678(22.3)$ & NA & \\
\hline Affective & $309(10.2)$ & NA & \\
\hline Pregnancy-related & $22(0.7)$ & NA & \\
\hline Year of the index date, median (IQR) & $2010(2007,2013)$ & $2010(2007,2013)$ & Matched \\
\hline Age, median (IQR) & $30(23,39)$ & $30(23,39)$ & Matched \\
\hline Male, $n(\%)$ & $1914(62.9)$ & $7656(62.9)$ & Matched \\
\hline Geographical region, $n(\%)$ & & & Matched \\
\hline London & $271(8.9)$ & $1084(8.9)$ & \\
\hline South England & $798(26.2)$ & $3192(26.2)$ & \\
\hline Midlands and East England & $599(19.7)$ & $2396(19.7)$ & \\
\hline North England & $532(17.5)$ & $2128(17.5)$ & \\
\hline Northern Ireland & $168(5.5)$ & $672(5.5)$ & \\
\hline Scotland & $356(11.7)$ & $1424(11.7)$ & \\
\hline Wales & $321(10.5)$ & $1284(10.5)$ & \\
\hline Smoking, $n(\%)$ & & & $<0.0001$ \\
\hline Non-smoker & $1011(33.2)$ & $6041(49.6)$ & \\
\hline Ever smoker & $1764(57.9)$ & $4031(33.1)$ & \\
\hline Unknown & $270(8.9)$ & $2108(17.3)$ & \\
\hline Alcohol consumption, $n(\%)$ & & & NS \\
\hline Non-drinker & $298(9.8)$ & $1026(8.4)$ & \\
\hline Ever drinker & $1742(57.2)$ & $5907(48.5)$ & \\
\hline Unknown & $1005(33.0)$ & $5247(43.1)$ & \\
\hline Body mass index, median (IQR) & $23.8(21.0,27.9)$ & $24.5(21.6,28.3)$ & $<0.0001$ \\
\hline$<18.5$ kg/m² (underweight), $n(\%)$ & $150(4.9)$ & $326(2.7)$ & \\
\hline$\geq 18.5 \mathrm{~kg} / \mathrm{m}^{2}$ and $<25 \mathrm{~kg} / \mathrm{m}^{2}$ (normal), $n(\%)$ & $1085(35.6)$ & $3578(29.4)$ & \\
\hline$\geq 25 \mathrm{~kg} / \mathrm{m}^{2}$ and $<30 \mathrm{~kg} / \mathrm{m}^{2}$ (overweight), $n(\%)$ & $501(16.5)$ & $2025(16.6)$ & \\
\hline$\geq 30$ kg/m² (obese), $n(\%)$ & $355(11.7)$ & $1355(11.1)$ & \\
\hline Unknown, $n(\%)$ & $954(31.3)$ & $4896(40.2)$ & \\
\hline $\begin{array}{l}\text { Number of different prescriptions in } 5 \text { years before } \\
\text { the index date, median (IQR) }\end{array}$ & $4(3,6)$ & $3(1,5)$ & $<0.0001$ \\
\hline $\begin{array}{l}\text { Number of GP consultations in } 5 \text { years before the } \\
\text { index date, median (IQR) }\end{array}$ & $55(29,95)$ & $25(10,53)$ & $<0.0001$ \\
\hline $4-5$ years prior to the index date & $6(2,15)^{+}$ & $3(1,9)$ & $<0.0001$ \\
\hline $3-4$ years prior to the index date & $7(2,16)^{\dagger}$ & $4(1,10)$ & $<0.0001$ \\
\hline $2-3$ years prior to the index date & $8(2,18)^{\dagger}$ & $4(1,11)$ & $<0.0001$ \\
\hline $1-2$ years prior to the index date & $9(3,20)^{\dagger}$ & $4(1,11)$ & $<0.0001$ \\
\hline $0-1$ year prior to the index date & $17(8,30)^{\dagger}$ & $4(1,12)$ & $<0.0001$ \\
\hline Number of symptom records in 5 years before the index date, median $(\mathrm{IQR})^{\ddagger}$ & $2(1,5)$ & $0(0,1)$ & $<0.0001$ \\
\hline
\end{tabular}

$p$ value obtained from chi-squared or from Mann-Whitney $U$ test as appropriate, and where applicable analysis excluded the 'unknown' category (missing data) FEP first episode psychosis, IQR interquartile range, NA not applicable, NS not significant

${ }^{+}$One-way ANOVA trend analysis within patients with FEP, $p<0.0001$

${ }^{\ddagger}$ Total number of coded records based on 55 studied individual symptoms 
consultations in the 12 months before diagnosis. The median number of recorded symptoms was 2 in FEP patients and 0 in controls over the 5 years (Table 2).

Respiratory comorbidity, musculoskeletal pain, and injury and major trauma were more common in FEP patients than in controls (Additional file 3).

\section{Prodrome symptoms}

Prevalence of recorded individual symptoms over the 5 years is given in Additional file 4.

Prevalence of all symptom groups was higher in FEP patients than in controls (Table 3). In patients with FEP, $48 \%$ were recorded to have at least one mood-related symptom in the 5 years prior to FEP diagnosis, compared to $11 \%$ of controls (adjusted OR 7.6 (95\% CI 6.8, 8.6)). 'Neurotic' symptoms, behavioural change, change in volition, and substance misuse were also frequently recorded $(>10 \%)$ in FEP patients. Perceptual problems, a typical psychotic symptom, were recorded in 5\% of FEP patients but hardly in any controls. Physical symptoms were recorded in $31 \%$ of FEP patients and $16 \%$ of controls (adjusted OR 1.8; 95\% CI 1.6, 2.0) (Table 3).

\section{Time from the first symptom to diagnosis}

Within each symptom group, the median time from first recorded symptom to diagnosis was around 2 to 2.5 years (range 732-975 days) although shorter for perceptual problems (70 days) (Additional file 5, Fig. 1a).

The earliest symptom, regardless of the symptom group, was recorded around 3 years (median 1065 days) before diagnosis (Additional file 6).

\section{Common symptom clusters in FEP patients}

The LCA analysis using recorded symptom groups prior to the diagnosis of FEP resulted in the three-cluster model providing the best fit based on BIC, CAIC, and bootstrap $p$ values (Additional file 7). FEP patients generally displayed high posterior probabilities for their assigned clusters, with mean posterior probabilities ranging from 0.71 to 0.81 across the three clusters (Table 4).

Based on the optimal model, 49\% $(n=1487)$ of FEP patients were in a cluster characterised by no or minimal symptoms recorded in primary care in the 5 years before diagnosis (the 'no or minimal symptom cluster', Fig. 1b). Forty-nine per cent $(734 / 1487)$ in this cluster had no prior recorded symptom, and $45 \%(669 / 1487)$ had presented with symptom(s) from only 1 symptom group, commonly a mood-related or physical symptom.

The second cluster contained 40\% $(n=1220)$ of FEP patients, and these had consulted with symptoms from at least 1 symptom group (median number 2), and patients in the cluster mainly had 'neurotic' $(74 \%)$ or mood-related (67\%) symptoms (the 'affective symptom cluster', Fig. 1c). Forty-four per cent (541/1220) of patients in this cluster had consulted for both moodrelated and 'neurotic' symptoms. All patients with a mood-related symptom in this cluster also presented with at least one other symptom from another symptom group.

Cluster 3 contained 11\% $(n=338)$ of FEP patients, and they consulted across multiple symptom groups (median number 4) with high probability of consulting in particular for physical (90\%), mood-related (90\%), 'neurotic' (69\%), behavioural (65\%), and volition change (62\%) symptoms (the 'multiple symptom cluster', Fig. 1d). All patients in cluster 3 consulted for symptoms in at least three symptom groups.

Relative to the affective symptom cluster, the no or minimal symptom cluster had a higher proportion of patients with non-affective psychosis, while the multiple symptom

Table 3 Five-year prevalence of symptom groups in patients prior to psychosis and matched participants

\begin{tabular}{|c|c|c|c|c|c|c|}
\hline \multirow[t]{2}{*}{ Symptom group } & \multicolumn{2}{|c|}{ FEP patients $(n=3045)$} & \multicolumn{2}{|c|}{ Matched participants $(n=12,180)$} & \multirow{2}{*}{$\begin{array}{l}\text { Crude odds } \\
\text { ratio }(95 \% \mathrm{Cl})^{\ddagger}\end{array}$} & \multirow{2}{*}{$\begin{array}{l}\text { Adjusted odds } \\
\text { ratio }^{\ddagger *}(95 \% \text { Cl) }\end{array}$} \\
\hline & $\overline{n^{\dagger}}$ & 5-year prevalence (\%) & $\overline{n^{\dagger}}$ & 5-year prevalence (\%) & & \\
\hline \multicolumn{7}{|l|}{ Psychological symptom } \\
\hline Mood-related symptom & 1473 & 48.4 & 1275 & 10.5 & $10.1(9.12,11.3)$ & $7.60(6.75,8.56)$ \\
\hline 'Neurotic' symptom & 1133 & 37.2 & 1106 & 9.1 & $6.54(5.89,7.27)$ & $4.87(4.35,5.47)$ \\
\hline Behavioural change & 490 & 16.1 & 657 & 5.4 & $3.53(3.08,4.05)$ & $2.67(2.30,3.09)$ \\
\hline Change in volition & 394 & 12.9 & 923 & 7.6 & $1.88(1.64,2.16)$ & $1.33(1.14,1.55)$ \\
\hline Perceptual problem & 162 & 5.3 & 5 & 0.04 & $130(53.7,313)$ & $112(44.4,283)$ \\
\hline Cognitive change & 38 & 1.3 & 30 & 0.3 & $5.41(3.35,8.75)$ & $3.80(2.04,7.09)$ \\
\hline Substance misuse & 338 & 11.1 & 134 & 1.1 & $13.0(10.4,16.4)$ & $8.09(6.36,10.3)$ \\
\hline Physical symptom & 939 & 30.8 & 1926 & 15.8 & $2.70(2.43,2.99)$ & $1.80(1.61,2.02)$ \\
\hline
\end{tabular}

FEP first episode psychosis, $\mathrm{Cl}$ confidence interval

${ }^{+}$Number of individuals with recorded symptom in the 5-year period before the index date

${ }^{*}$ Conditional logistic regression analyses with cluster-robust variance estimator

*Adjusted for smoking, alcohol consumption, BMI morbidity burden, and specific co-morbid conditions (including respiratory condition, musculoskeletal pain, and injury and major trauma), in addition to matched year of birth (age), gender, and practice 
All patients, $n=3045$ (a)

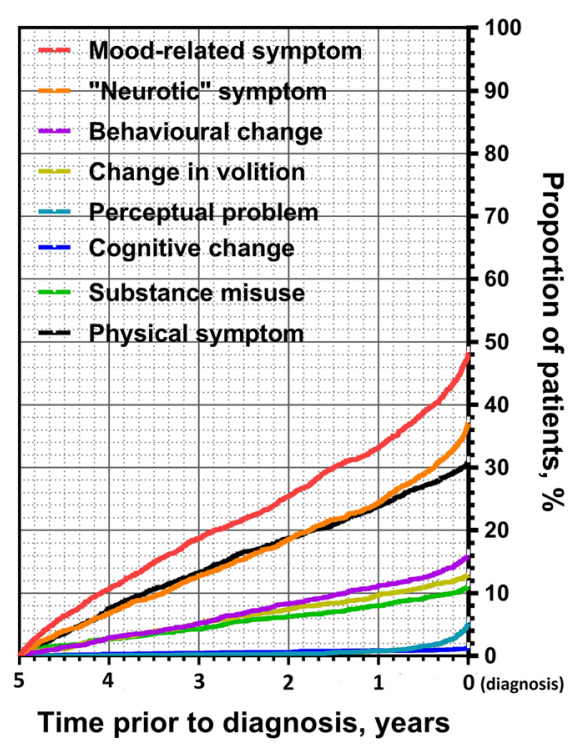

Patients in ASC, $n=1220$ (c)

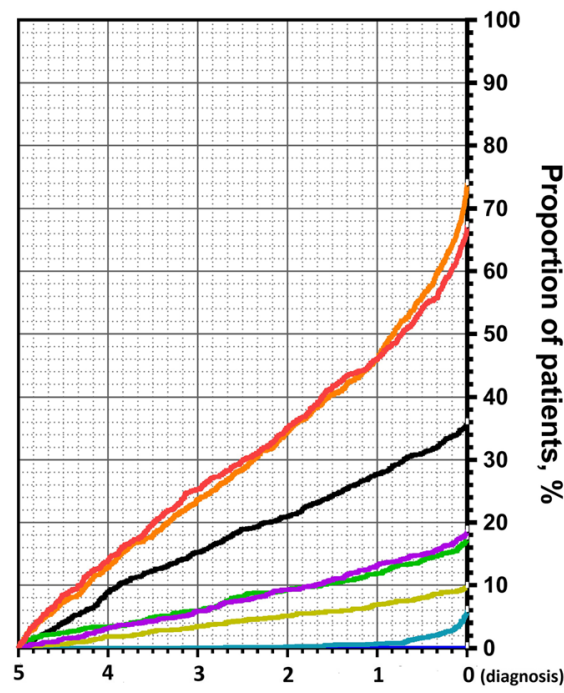

Time prior to diagnosis, years
Patients in NMSC, $n=1487$ (b)

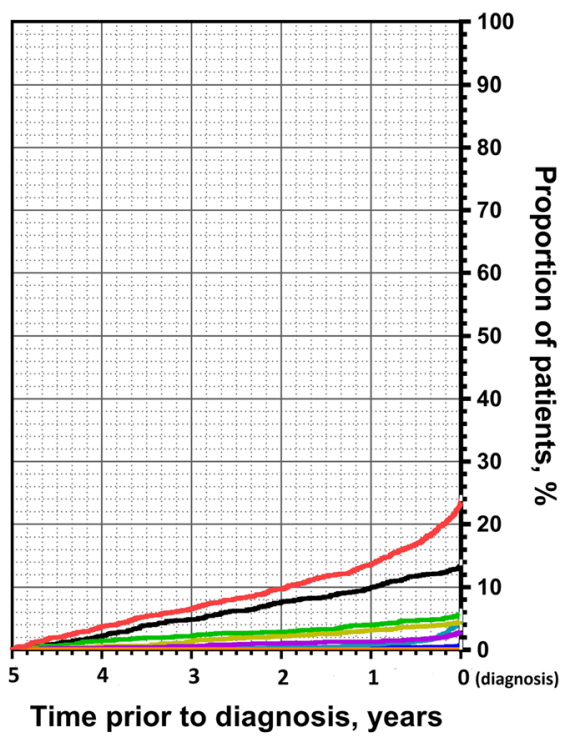

Patients in MSC, $n=338$ (d)

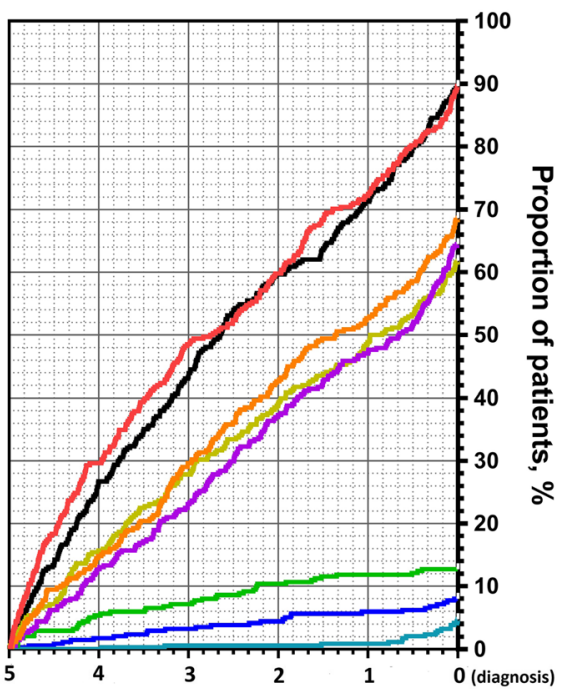

Time prior to diagnosis, years

Fig. 1 Cumulative proportion of patients having a prodrome symptom recorded in primary care from 5 years before until the time of FEP diagnosis. FEP, first episode psychosis; NMSC, no or minimal symptom cluster; ASC, affective symptom cluster; MSC, multiple symptom cluster

cluster included a higher proportion of patients with affective and drug-induced psychosis (Table 5). The no or minimal symptom cluster patients were the youngest (median age 29 years) and had the highest percentage being male (72\%). The multiple symptom cluster patients were the oldest (median age 33 years) and had the lowest percentage being male (36\%). This cluster also had the highest rates of recorded obesity, alcohol drinking, morbidity burden, and frequency of GP consultations (Table 5). Patients in the multiple symptom cluster had a median of 4 years (median 1548 days) from earliest symptom to diagnosis, with mood-related (31\%) and physical (29\%) as the most common earliest symptoms (Additional file 6).

\section{Symptom clusters emerging over time before FEP diagnosis}

Nine per cent of FEP patients could already be classified into the affective symptom cluster at 4 years before diagnosis and $28 \%$ at 1 year before diagnosis. One per cent of FEP patients could be classified in the multiple symptom cluster 4 years before diagnosis, rising to $7 \%$ at 1 year before diagnosis (Fig. 2). 
Table 4 Cluster classification: posterior probability of membership of clusters

\begin{tabular}{llll}
\hline $\begin{array}{l}\text { Assigned cluster, } \\
n(\%)\end{array}$ & \multicolumn{3}{l}{ Mean posterior probability for each cluster } \\
\cline { 2 - 4 } $\begin{array}{l}\text { NMSC, } n=1487 \\
(48.8)\end{array}$ & 0.809 & ASC & MSC \\
$\begin{array}{l}\text { ASC, } n=1220 \\
(40.1)\end{array}$ & 0.128 & 0.174 & 0.018 \\
$\begin{array}{l}\text { MSC, } n=338 \\
(11.1)\end{array}$ & 0.022 & 0.713 & 0.159 \\
\hline
\end{tabular}

NMSC no or minimal symptom cluster, ASC affective symptom cluster, MSC multiple symptom cluster

\section{Discriminative ability of symptom clusters on FEP diagnosis}

The majority of controls $(87 \%, n=10,554)$ were mapped to the no or minimal symptom cluster, followed by the affective (11\%, $n=1314)$ and multiple $(3 \%, n=312)$ symptom clusters. The affective and multiple symptom clusters (versus the no or minimal symptom cluster) demonstrated a good discriminative ability for FEP predictive classification (C-statistic 0.766 (95\% CI 0.757, $0.775)$; sensitivity $=51.2 \%$; specificity $=86.7 \%$ ).

\section{Discussion}

This large study utilising a national primary care database has shown three distinct patterns of symptom presentation prior to FEP diagnosis, with more than one in ten patients presenting with multiple different symptoms in the 5 years prior to diagnosis. Many patients diagnosed with FEP have a long history of relevant symptom presentation to primary care.

\section{Major findings}

This study has shown that symptoms are often presented several years prior to the diagnosis of FEP. The median time interval between the first recorded potential prodromal symptom and a coded FEP diagnosis was around two or more years. This is longer than the reported average DUP [2-4], although our study in primary care cannot assess whether the recorded symptoms are part of the FEP prodrome.

In particular, there are a group of patients $(11 \%$ of all those diagnosed with FEP) who have presented with multiple symptoms in the previous 5 years before diagnosis. This group has high rates of consultations and present with a range of morbidity before the diagnosis of FEP. This group may represent patients who could be recognised sooner as the median time of the earliest recorded symptom is about 4 years before FEP. Approximately two thirds of this cluster were female, and it may be that GPs are less likely to recognise potential FEP in females. The second group of patients (40\%) mainly presented with affective symptoms (such as moodrelated and neurotic symptoms) prior to diagnosis. The third group of patients (49\%) had no or minimal symptoms recorded in primary care, suggesting either an insidious onset disease or limited prior use of primary care. The group tended to be of younger age at diagnosis, male, with lower BMI, morbidity burden, and lower rate of GP consultations.

We found that many patients who later go on to receive the diagnosis of FEP consulted their GP with increasing regularity over the 5 years before diagnosis and particularly in the 12 months before diagnosis (more than quadruple the frequency of consultation than our control group). These findings confirm this population in general is actively help-seeking, but those prodromal symptom presentations may be difficult for GPs to elicit and distinguish from less severe states and disorders. However, there remains a quarter of patients with no record of coded symptoms in the primary care database, who may not be actively seeking related help from their GP in the 5 years before FEP diagnosis.

High rates of recorded physical co-morbidities such as respiratory conditions might be expected in a population known to smoke heavily [25]. The high rates of recorded physical symptoms, and with comorbid musculoskeletal pain, are a novel finding and may relate to the increased levels of somatic complaints prior to FEP diagnosis. Somatic complaints have previously been recognised to be a frequent manifestation of psychological distress in common mental health disorders such as depression and anxiety [26].

Mood-related, 'neurotic', and physical symptoms were among the most frequently recorded. Common psychological symptoms that characterise the at risk mental state or the ultrahigh risk stage before the actual diagnosis of psychosis include social isolation or withdrawal, impairment in personal hygiene and grooming, blunted or inappropriate affect, odd beliefs or magical thinking, and unusual perceptual experiences [27]. These were not commonly recorded symptoms in primary care, but once coded, heralded a shorter period to diagnosis. GPs may be reluctant to enquire about psychotic symptoms. It is possible that the low prevalence of these symptoms in the GP records is due to inadequate awareness that these clinical manifestations may herald the onset of psychosis.

Substance misuse was commonly recorded in FEP patients. This underlines the importance of GPs recognising that FEP is commonly preceded or accompanied by co-morbid substance misuse. It is therefore important not to misattribute relevant symptom presentations purely to substance misuse without careful exploration of the possibility of an emerging psychotic disorder [28]. 
Table $\mathbf{5}$ Characteristics at diagnosis of FEP patients by cluster

\begin{tabular}{|c|c|c|c|c|}
\hline & Symptom clusters & & & $p$ value $^{\dagger}$ \\
\hline & $\begin{array}{l}\text { NMSC patients } \\
(n=1487)\end{array}$ & $\begin{array}{l}\text { ASC patients } \\
(n=1220)\end{array}$ & $\begin{array}{l}\text { MSC patients } \\
(n=338)\end{array}$ & \\
\hline Type of psychosis, $n(\%)^{\ddagger}$ & & & & $<0.0001$ \\
\hline Non-affective & $1023(68.8)$ & $819(67.1)$ & $194(57.4)$ & \\
\hline Drug-induced & $305(20.5)$ & $277(22.7)$ & $96(28.4)$ & \\
\hline Affective & $146(9.8)$ & $117(9.6)$ & $46(13.6)$ & \\
\hline Male, $n(\%)$ & $1064(71.6)$ & $727(59.6)$ & $123(36.4)$ & $<0.0001$ \\
\hline Age, median (IQR) & $29(21,38)$ & $31(24,38)$ & $33(25,41)$ & $<0.0001$ \\
\hline Year of the index date, median (IQR) & $2010(2007,2013)$ & $2010(2007,2013)$ & $2010(2007,2012)$ & NS \\
\hline Geographical region, $n(\%)$ & & & & $<0.0001$ \\
\hline London & $158(10.6)$ & $92(7.5)$ & $21(6.2)$ & \\
\hline South England & $406(27.3)$ & $300(24.6)$ & $92(27.2)$ & \\
\hline Midlands and East England & $274(18.4)$ & $249(20.4)$ & $76(22.5)$ & \\
\hline North England & $243(16.3)$ & $236(19.3)$ & $53(15.7)$ & \\
\hline Northern Ireland & $53(3.6)$ & $92(7.5)$ & $23(6.8)$ & \\
\hline Scotland & $189(12.7)$ & $125(10.3)$ & $42(12.4)$ & \\
\hline Wales & $164(11.0)$ & $126(10.3)$ & $31(9.2)$ & \\
\hline Smoking, $n(\%)$ & & & & 0.022 \\
\hline Non-smoker & $495(33.3)$ & $387(31.7)$ & $129(38.2)$ & \\
\hline Ever smoker & $802(53.9)$ & $768(63.0)$ & $194(57.4)$ & \\
\hline Unknown & $190(12.8)$ & $65(5.3)$ & $15(4.4)$ & \\
\hline Alcohol consumption, $n(\%)$ & & & & 0.005 \\
\hline Non-drinker & $155(10.4)$ & $112(9.2)$ & $31(9.2)$ & \\
\hline Ever drinker & $730(49.1)$ & $777(63.7)$ & $235(69.5)$ & \\
\hline Unknown & $602(40.5)$ & $331(27.1)$ & $72(21.3)$ & \\
\hline Body mass index, median (IQR) & $23.4(20.9,27.1)$ & $23.8(21.0,28.1)$ & $25.0(21.3,30.2)$ & $<0.0001$ \\
\hline$<18.5 \mathrm{~kg} / \mathrm{m}^{2}$ (underweight), $n(\%)$ & $65(4.4)$ & $61(5.0)$ & $24(7.1)$ & \\
\hline $\begin{array}{l}\geq 18.5 \mathrm{~kg} / \mathrm{m}^{2} \text { and }<25 \mathrm{~kg} / \mathrm{m}^{2} \text { (normal), } \\
\text { n (\%) }\end{array}$ & $502(33.8)$ & $460(37.7)$ & $123(36.4)$ & \\
\hline $\begin{array}{l}\geq 25 \mathrm{~kg} / \mathrm{m}^{2} \text { and }<30 \mathrm{~kg} / \mathrm{m}^{2} \text { (overweight), } \\
n(\%)\end{array}$ & $215(14.5)$ & $215(17.6)$ & $71(21.0)$ & \\
\hline$\geq 30 \mathrm{~kg} / \mathrm{m}^{2}$ (obese), $n(\%)$ & $125(8.4)$ & $154(12.6)$ & $76(22.5)$ & \\
\hline Unknown, $n(\%)$ & $580(39.0)$ & $330(27.1)$ & $44(13.0)$ & \\
\hline $\begin{array}{l}\text { Number of different prescriptions in } 5 \text { years } \\
\text { before diagnosis, median (IQR) }\end{array}$ & $3(2,5)$ & $5(3,7)$ & $7(5,8)$ & $<0.0001$ \\
\hline $\begin{array}{l}\text { Number of GP consultations in } 5 \text { years before } \\
\text { diagnosis, median (IQR) }\end{array}$ & $36(19,65)$ & $66(42,103)$ & $119(79,171)$ & $<0.0001$ \\
\hline $4-5$ years prior to diagnosis & $4(1,10)$ & $8(3,17)$ & $15(8,27)$ & $<0.0001$ \\
\hline $3-4$ years prior to diagnosis & $4(1,11)$ & $9(3,18)$ & $18(9,33)$ & $<0.0001$ \\
\hline $2-3$ years prior to diagnosis & $5(1,11)$ & $10(4,20)$ & $21(11,33)$ & $<0.0001$ \\
\hline $1-2$ years prior to diagnosis & $6(2,13)$ & $13(5,22)$ & $21(12,40)$ & $<0.0001$ \\
\hline $0-1$ year prior to diagnosis & $11(5,21)$ & $21(11,33)$ & $32(19,52)$ & $<0.0001$ \\
\hline
\end{tabular}

FEP first episode psychosis, NMSC no or minimal symptom cluster, ASC affective symptom cluster, MSC multiple symptom cluster, NS not significant ${ }^{+}$Obtained from chi-squared or univariable multinomial logistic regression analysis as appropriate, and where applicable analysis excluded the unknown category (missing data)

${ }^{\ddagger}$ Data were not reported for pregnancy-related psychosis due to CPRD reporting policy that no cell should contain fewer than five events 


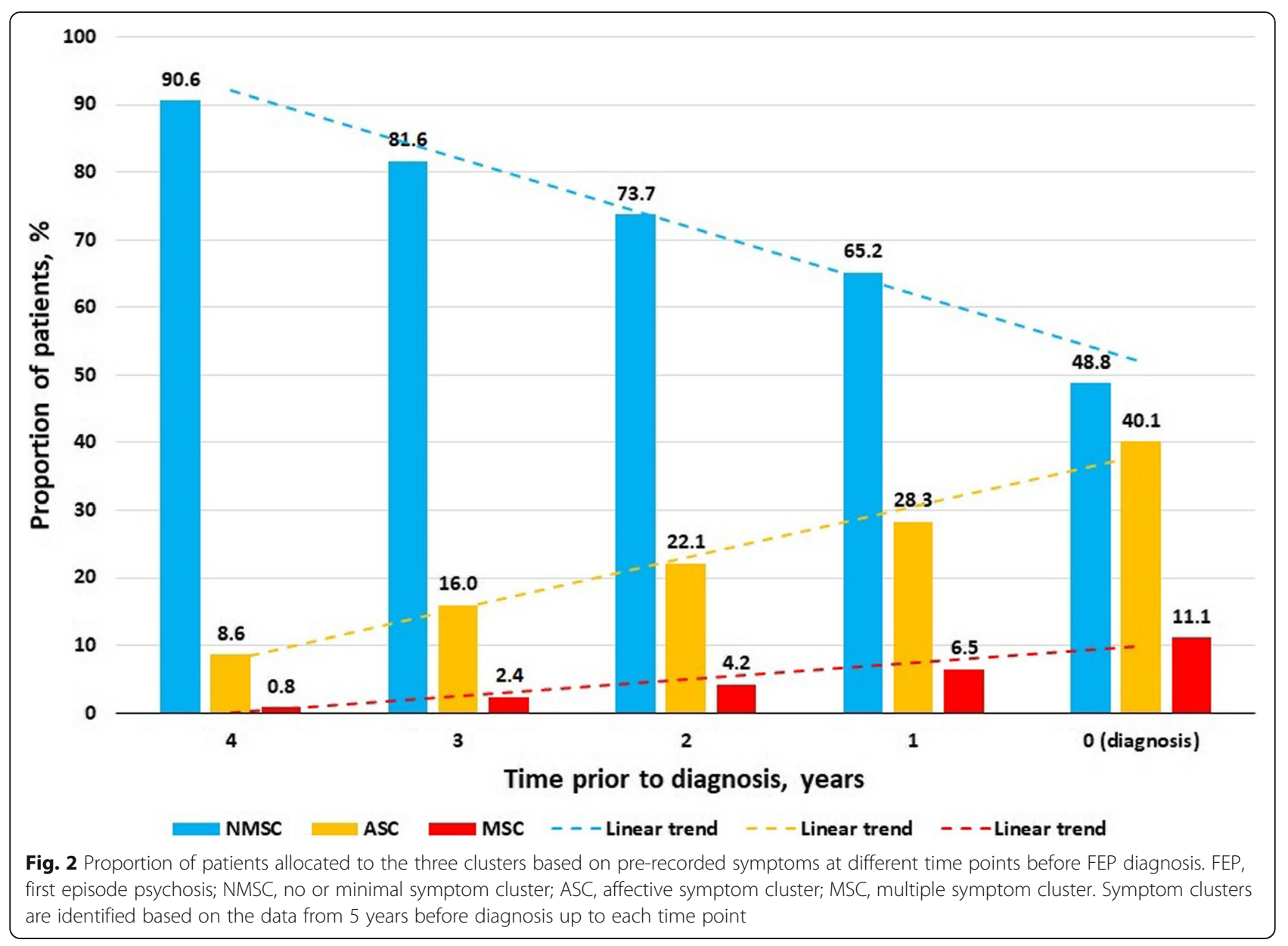

The affective and multiple symptom cluster patterns were rare in our control group. Our discriminative ability analysis for FEP classification has shown that these two patterns of symptom presentation are suggestive (C-statistic 0.766) for a potential FEP. Importantly, many patients already had these symptom patterns several years before a FEP diagnosis (one third 1 year before diagnosis, a quarter at 2 years before diagnosis, a fifth at 3 years before diagnosis), giving an opportunity for earlier awareness, referral, and diagnosis.

\section{Comparison with the existing literature}

The literature on the patterns of symptoms in the potentially long prodromal period before the onset of psychosis is limited. Most studies on the identification of symptom patterns focus on help-seeking ultrahigh-risk populations in specialised mental health service settings [29-31] or examined individual symptoms prior to the diagnosis [32]. We are not aware of any other previous study that has investigated the pattern of symptoms presentation for individuals who are help-seeking in primary care over a long observational period prior to diagnosis, although a survey investigation has modelled subtypes of psychosis-like experiences in the general population using similar latentclass analytical approach [33].

An American study described patterns of health care use before FEP in adolescents and young adults. Although it included previous diagnoses of depressive, anxiety, attention deficit, bipolar, and substance use disorders as an indication of mental health problem, the pattern of symptoms and their relationship with the diagnosis of FEP was not studied [34]. Sullivan et al. examined 13 individual symptoms recorded in primary care to assess the positive predictive value of single or paired symptoms for a diagnosis of psychosis between 2000 and 2009 [32]. The majority of the symptoms $(n=12)$ were linked to later psychosis diagnosis, with suicidal behaviour as the most strongly associated common predictor. We also found a strong association with suicidal behaviour and self-harm but have extended Sullivan's study by examining 55 individual symptoms and determining common patterns of presentation prior to diagnosis. 


\section{Strengths and limitations}

The increasing availability and quality of routinely recorded longitudinal primary care electronic health records offer the opportunity to investigate the patterns of symptoms recorded before a FEP diagnosis in a larger and more generalizable setting. We restricted the analysis to the period following the introduction of the QOF in the UK which increased the quality of recording in primary care. However, the recording of diagnosis of FEP made in secondary care may be delayed in entry into primary care records, and patients with a diagnosis of psychosis recorded only in secondary care would not be included in our analysis. We excluded those with a long history of antipsychotic medication (more than 1 year) which should mean patients with FEP included in our analysis had a recent diagnosis, and this study used a UK national primary care database (CPRD) with previous validation of the accuracy and completion of diagnosis recording of mental and behavioural disorders [13]. The median age (30 years old) at FEP diagnosis in our study was comparable with that (31) at a treated incidence of psychotic disorder in a recent large European (including England) multinational study [35]. A 5-year observational period was considered long enough to identify insidious symptoms related to FEP, although it is possible that relevant symptoms will present more than 5 years before FEP diagnosis. Not all symptoms identified will necessarily have reflected a prodromal phase of FEP in all patients. A further limitation to this study is the lack of primary care recording of concerns raised by families, potentially an important factor in alerting a GP to the presence of a more serious mental disorder. We included over 50 individual symptoms identified through a review of the literature [17-19], but other symptoms may be missed. We summarised the psychological symptoms into 6 groups largely based on the work of Yung and McGorry [17], but there may be other approaches to grouping the symptoms. We included 15 physical symptoms together as a single group, but we are aware that these somatic complaints are diverse in nature. It is possible that symptoms may not be coded but are recorded in the consultation free text that GPs use alongside the Read codes. However, it is likely that those with a coded symptom are those with more troublesome or noticeable symptoms. No attempt was made to grade the degree of each symptom. There was some missing data on covariates including smoking, alcohol drinking, and BMI, as is usual in research using primary care health record databases and particularly in younger populations. The rapid development of the early intervention in psychosis services in England between 2000 and 2010 may have caused geographical variation in the responsiveness of GPs to psychosis presentations where they had direct access to specialist FEP assessment [36]. The generalisability of the findings from this study has yet to be assessed.

\section{Impact and implications}

The criteria that use psychiatric and cognitive symptoms for recognition of prodromal stage or at risk mental state for psychosis do not work well outside defined clinical population samples and not within the general population seeking help in primary care [37]. This study highlights the opportunities in primary care for identifying patients who may be experiencing a prodromal state of psychosis rather than awaiting the emergence of major psychotic symptoms or acute psychosis. Our study has highlighted there is a significant minority (often female) who may be waiting several years for a diagnosis and are actively seeking help in primary care. This is a group whose characteristics GPs should be particularly be aware of to allow the opportunity for earlier recognition, referral, and diagnosis. The three clusters suggested in the present study represent the first attempt to link common prodromal presentations in primary care to the identification of subsequent FEP. The curriculum and training programmes for GPs need a greater focus on early detection of symptoms suggestive of psychosis and its prodrome. It may be possible to link these symptoms and clusters with existing criteria and other markers (such as increasing frequency of GP attendance, suicidality, social withdrawal, and a history of severe mental illness) to identify the people at high risk of psychosis at the earliest possible stage, as a risk prediction model, hence contributing to more effective treatment strategies to improve outcomes.

\section{Patient and public involvement}

The findings were discussed with a convenor of a local support group ('Hear Our Voices', North Staffordshire, England) that aims to give a voice to people with a mental health problem. He suggested that the findings made sense and emphasised the reluctance of men, who might use alcohol and cigarettes as a coping strategy, to access care. He suggested that the association of lower BMI with FEP patients may reflect a lack of self-care despite possible metabolic abnormalities, and the observation of increased injury and major trauma in FEP patients may be due to excess risky behaviours. He was concerned that suicidality was recorded in about $6 \%$ of people who were later diagnosed with FEP, about 14 times commoner than in controls. He emphasised the importance of this work in providing clues for GPs to increase their awareness of the possibility of an emerging psychosis.

\section{Conclusions}

Our study identified three distinctive patterns of prodromal symptom presentations in patients seeking help in primary care and subsequently diagnosed with FEP. Awareness of these symptom clusters may help GPs to identify patients who are experiencing a prodromal state of psychosis, thereby facilitating more timely access to specialist assessment and treatment and hence better long-term outcomes. 


\section{Supplementary information}

Supplementary information accompanies this paper at https://doi.org/10. 1186/s12916-019-1462-y.

Additional file 1. Comparison of patient characteristics and patterns of symptoms in FEP patients with and without missing data on smoking, alcohol drinking and BMI.

Additional file 2. Definitions (psychosis, prodromal symptom and antipsychotic medication).

Additional file 3. Comorbid conditions measured at index date in FEP patients and matched participants.

Additional file 4. Five-year prevalence of prodrome symptoms in FEP patients and matched participants.

Additional file 5. Time interval between first symptom (if any) and FEP diagnosis within each symptom group.

Additional file 6. Profile of the earliest symptom, among all symptom groups, in patients with FEP.

Additional file 7. Statistical assessment of the optimal number of clusters from latent class analysis models based on eight groups of prodrome symptoms.

\section{Abbreviations}

BIC: Bayes Information Criterion; BMI: Body mass index; BNF: British National Formulary; CAIC: Consistent Akaike's Information Criterion; Cl: Confidence interval; CPRD: Clinical Practice Research Datalink; DUP: Duration of untreated psychosis; FEP: First episode psychosis; GP: General practitioner; LCA: Latent class analysis; OR: Odds ratio; QOF: Quality and Outcomes Framework

\section{Acknowledgements}

This study is based in part on the data from the Clinical Practice Research Datalink obtained under licence from the UK Medicines and Healthcare products Regulatory Agency. The data is provided by patients and collected by the NHS as part of their care and support. The interpretation and conclusions contained in this study are those of the authors alone. We would like to thank Adam Colclough, from 'Hear Our Voices', North Staffordshire, England, for his input into the interpretation of the findings and for commenting on the draft manuscript.

\section{Authors' contributions}

SF, KJ, and JE conceived the study. YC analysed the data and drafted the initial manuscript. All authors contributed to the study design and interpretation of the data, and all authors approved the final version of the manuscript submitted for publication.

\section{Funding}

This paper presents independent research funded by the Royal College of General Practitioners (RCGP) Scientific Foundation Board grant (SFB-2017-13). The views expressed are those of the authors.

\section{Availability of data and materials}

No additional data or material available if not stated elsewhere.

\section{Ethics approval and consent to participate}

This study was approved by the CPRD Independent Scientific and Advisory Committee (ISAC, Protocol No. 18_026).

\section{Consent for publication}

Not applicable.

\section{Competing interests}

DS is an expert advisor to the NICE Centre for guidelines, a member of the current NICE Guideline Development Group for Rehabilitation in adults with complex psychosis and related severe mental health conditions, and a board member of the National Collaborating Centre for Mental Health (NCCMH); the views are personal and not those of NICE or NCCMH. The other authors declare that they have no competing interests.

\section{Author details}

School of Primary, Community and Social Care, Keele University, Keele ST5 5BG, UK. ${ }^{2}$ University of Manchester, Manchester M13 9PL, UK. ${ }^{3}$ Psychosis Research Unit, Greater Manchester Mental Health NHS Trust, Manchester M25 3BL, UK. ${ }^{4}$ School of Pharmacy, Keele University, Keele ST5 5BG, UK.

Received: 11 April 2019 Accepted: 5 November 2019

Published online: 04 December 2019

\section{References}

1. Perkins DO, Gu H, Boteva K, Lieberman JA. Relationship between duration of untreated psychosis and outcome in first-episode schizophrenia: a critical review and meta-analysis. Am J Psychiatry. 2005;162:1785-804.

2. Large M, Farooq S, Nielssen O, Slade T. Relationship between gross domestic product and duration of untreated psychosis in low- and middleincome countries. Br J Psychiatry. 2008;193:272-8.

3. Souaiby L, Gaillard R, Krebs MO. Duration of untreated psychosis: a state-ofthe-art review and critical analysis. Encephale. 2016:42:361-6.

4. Penttilä $M$, Jääskeläinen $E$, Hirvonen $N$, Isohanni $M$, Miettunen J. Duration of untreated psychosis as predictor of long-term outcome in schizophrenia: systematic review and meta-analysis. Br J Psychiatry. 2014;205:88-94.

5. Marshall M, Lewis S, Lockwood A, Drake R, Jones P, Croudace T. Association between duration of untreated psychosis and outcome in cohorts of firstepisode patients. Arch Gen Psychiatry. 2005:62:975-83.

6. Lloyd-Evans B, Crosby M, Stockton S, Pilling S, Hobbs L, Hinton M, et al. Initiatives to shorten duration of untreated psychosis: systematic review. $\mathrm{Br}$ J Psychiatry. 2011;198:256-63.

7. Anderson KK, Further R, Malla AK. The pathways to mental health care of first-episode psychosis patients: a systematic review. Psychol Med. 2010;40:1585-97.

8. Skeate A, Jackson C, Birchwood M, Jones C. Duration of untreated psychosis and pathways to care in first-episode psychosis. Investigation of helpseeking behaviour in primary care. Br J Psychiatry. 2002;43:s73-7.

9. Platz C, Umbricht DS, Cattapan-Ludewig K, Dvorsky D, Arbach D, Brenner HD, et al. Help-seeking pathways in early psychosis. Soc Psychiatry Psychiatr Epidemiol. 2006;41:967-74

10. Cratsley K, Regan J, McAllister V, Simic M, Aitchison KJ. Duration of untreated psychosis, referral route, and age of onset in an early intervention in psychosis service and a local CAMHS. Child Adolesc Mental Health. 2008;13:130-3.

11. Nørgaard $H$, Søndergaard Pedersen $H$, Fenger-Grøn M, Mors O, Nordentoft $M$, Vestergaard $M$, et al. Increased use of primary care during 6 years of prodromal schizophrenia. Acta Psychiatr Scand. 2016;134:225-33.

12. Herrett E, Gallagher AM, Bhaskaran K, Forbes H, Mathur R, van Staa T, et al Data resource profile: Clinical Practice Research Datalink (CPRD). Int J Epidemiol. 2015;44:827-36.

13. Herrett E, Thomas SL, Schoonen WM, Smeeth L, Hall AJ. Validation and validity of diagnoses in the General Practice Research Database: a systematic review. Br J Clin Pharmacol. 2010;69:4-14.

14. Hardoon S, Hayes JF, Blackurn R, Petersen I, Walters K, Nazareth I, et al. Recording of severe mental illness in United Kingdom primary care, 20002010. PLoS One. 2013;8(12):e82365. https://doi.org/10.1371/journal.pone. 0082365. eCollection 2013.

15. Quality and Outcomes Framework. http://content.digital.nhs.uk/qof. Accessed 23 Aug 2019.

16. Hennessy S, Bilker WB, Berlin JA, Strom BL. Factors influencing the optimal control-to-case ratio in matched case-control studies. Am J Epidemiol. 1999; 149:195-7.

17. Yung AR, McGorry PD. The prodromal phase of first-episode psychosis: past and current conceptualizations. Schizophr Bull. 1996;22:353-70.

18. Larson MK, Walker EF, Compton MT. Early signs, diagnosis and therapeutics of the prodromal phase of schizophrenia and related psychotic disorders. Expert Rev Neurother. 2010:10:1347-59.

19. Jackson HJ, McGorry PD, Dudgeon P. Prodromal symptoms of schizophrenia in first-episode psychosis: prevalence and specificity. Compr Psychiatry. 1995;36:241-50

20. Smith DJ, Langan J, McLean G, Guthrie B, Mercer SW. Schizophrenia is associated with excess multiple physical-health comorbidities but low levels of recorded cardiovascular disease in primary care: cross-sectional study. BMJ Open. 2013;3:e002808 
21. Brilleman SL, Salisbury C. Comparing measures of multimorbidity to predict outcomes in primary care: a cross sectional study. Fam Pract. 2013;30:172-8.

22. Stuart-Buttle CD, Read JD, Sanderson HF, Sutton YM. A language of health in action: Read codes, classification and groupings. Proc AMIA Fall Symp. 1996;1996:75-9.

23. Collins LM, Lanza ST. Latent class and latent transition analysis: with applications in the social, behavioural, and health sciences. Hoboken, New Jersey: Wiley; 2005.

24. Nagin DS. Group-based modeling of development. Cambridge: Harvard University Press; 2005.

25. Carney R, Cotter J, Bradshaw T, Firth J, Yung AR. Cardiometabolic risk factors in young people at ultra-high risk for psychosis: a systematic review and meta-analysis. Schizophr Res. 2016;170:290-300.

26. Bridges KW, Goldberg DP. Somatic presentation of DSM-III psychiatric disorders in primary care. J Psychosom Res. 1987;29:563-9.

27. Tandon N, Shah J, Keshavan MS, Tandon R. Attenuated psychosis and the schizophrenia prodrome: current status of risk identification and psychosis prevention. Neuropsychiatry. 2012;2:345-53.

28. Frisher M, Martino Ol, Bashford J, Crome I, Croft P. Incidence of psychoses among drug dependent patients in primary care with no psychiatric history: a retrospective observational matched-cohort study. Eur J Psychiat. 2013;27:240-7.

29. Healey KM, Penn DL, Perkins D, Woods SW, Keefe RSE, Addington J. Latent profile analysis and conversion to psychosis: characterizing subgroups to enhance risk prediction. Schizophr Bull. 2018;44:286-96.

30. Velthorst E, Derks EM, Schthorst P, Becker H, Durston S, Ziermans T, et al. Quantitative and qualitative symptomatic differences in individuals at ultrahigh risk for psychosis and healthy controls. Psychiatry Res. 2013;210:432-7.

31. Ryan AT, Addington J, Bearden CE, Cadenhead KS, Cornblatt BA, Mathalon $\mathrm{DH}$, et al. Latent class cluster analysis of symptom ratings identifies distinct subgroups within the clinical high risk for psychosis syndrome. Schizophr Res. 2018;197:522-30.

32. Sullivan SA, Hamilton W, Tilling K, Redaniel T, Moran P, Lewis G. Association of primary care consultation patterns with early signs and symptoms of psychosis. JAMA Netw Open. 2018;1:e185174.

33. Shevlin M, Murphy J, Dorahy MJ, Adamson G. The distribution of positive psychosis-like symptoms in the population: a latent class analysis of the National Comorbidity Survey. Schizophr Res. 2007;89:101-9.

34. Simon GE, Stewart C, Hunkeler EM, Yarborough BJ, Lynch F, Coleman KJ et al. Care pathways before first diagnosis of a psychotic disorder in adolescents and young adults. Am J Psychiatry. 2018;175:434-42.

35. Jongsma HE, Gayer-Anderson C, Lasalvia A, Quattrone D, Mulè A, Szöke A, et al. The treated incidence of psychotic disorders in the multinational EUGEl study. JAMA Psychiatry. 2018;75:36-46.

36. National Service Framework for Mental Health. London: Department of Health; 1999.

37. Fusar-Poli P. Why ultra high risk criteria for psychosis prediction do not work well outside clinical samples and what to do about it. World Psychiatry. 2017;16:212-3.

\section{Publisher's Note}

Springer Nature remains neutral with regard to jurisdictional claims in published maps and institutional affiliations.

\section{Ready to submit your research? Choose BMC and benefit from:}

- fast, convenient online submission

- thorough peer review by experienced researchers in your field

- rapid publication on acceptance

- support for research data, including large and complex data types

- gold Open Access which fosters wider collaboration and increased citations

- maximum visibility for your research: over $100 \mathrm{M}$ website views per year

At $\mathrm{BMC}$, research is always in progress.

Learn more biomedcentral.com/submissions 